\title{
Shifted Legendre Polynomials algorithm used to the dynamic analysis of viscoelastic pipes conveying fluid with the variable fractional order model th
}

\author{
Yuanhui Wang ${ }^{\mathrm{a}}$, Yiming Chen ${ }^{\mathrm{a}, \mathrm{b}, \mathrm{c}, *}$ \\ ${ }^{a}$ College of Sciences, Yanshan University, Qinhuangdao, 066004, Hebei, China \\ ${ }^{b}$ LE STUDIUM RESEARCH FELLOW, Loire Valley Institute for Advanced Studies \\ Orléans \& Tours, France \\ ${ }^{c}$ PRISME (INSA-Institut National des sciences appliqués)-88, Boulevard Lahitolle \\ 18000 Bourges, France
}

\begin{abstract}
The dynamic analysis of viscoelastic pipes conveying fluid is investigated with the variable fractional order model in this article. The nonlinear variable fractional order integral-differential equation is established by introducing the model into the governing equation. Then the Shifted Legendre Polynomials algorithm is first presented for dealing with this kind of equations. The numerical example verifies that the algorithm is an effective and accurate technique for addressing this type complicated equation. Numerical results for dynamic analysis of viscoelastic pipes conveying fluid show the effect of parameters on displacement, acceleration, strain and stress. It also indicates that how dynamic properties are affected by the variable fractional order and fluid velocity varying. Most of all, the proposed algorithm has enormous potentials for the problem of high precision dynamics with the variable fractional order model.

Keywords: Variable fractional order model, Dynamic analysis, Viscoelastic pipes, Nonlinear integral-differential equation, Shifted Legendre Polynomials algorithm, Chaotic motion
\end{abstract}

\footnotetext{
${ }^{\text {W }}$ Fully documented templates are available in the elsarticle package on CTAN

* Corresponding author

Email address: chenym@ysu.edu.cn (Yiming Chen)
}

Preprint submitted to Applied Mathematical Modelling 


\section{Introduction}

Pipes conveying fluid, as the important structures, have been paid lots of attentions from both engineering and science point of view. Considering the impact of the external and internal factors, changes for the dynamic nature of the pipes will occur, which would lead to the uncertainty of pipes conveying fluid on engineering performance.

According to the aforementioned reason, many remarkable achievements on dynamic analysis of pipes conveying fluid are available in [1] 8 . The dynamic analysis of inclined supported pipes conveying fluid was addressed in [1]. The article 2] researched cantilevered pipes in the presence of a periodic force excitation. By virtual of a nonlinear equation, the responses of pipes were discussed under various conditions. Elastic and slender pipes conveying fluid were studied under a top end force excitation and uniform cross flowing in 3 . The research results showed the internal external factors have critical effects on inhibiting vortex-induced vibrations. In [4, the influences of flow velocity on the stabilization problems of pipes conveying fluid were investigated. However, the purpose of the aforementioned studies was to analyze the stability problem. It is the first time that chaotic dynamics of cantilevered pipes conveying pulsating fluid with the external load were studied [5. It is noted that there are great dangers to cause the chaotic vibrations for the mentioned pipes. Dynamics of the external and internal resonance on pipes conveying fluid were addressed in [6] under nonlinear forced vibration. Some physical and geometrical quantities were introduced in [7, and they were studied in how to influence dynamics properties. The non-linear vibration for viscoelastic pipes conveying fluid was investigated based on a fractional order model in 8 . A mount of works have been done, but still exist some issues needed to be further considered for pipes conveying fluid.

Viscoelastic materials have the widespread application in pipes conveying fluid due to its damping and simulating biological tissue. Pipes with viscoelastic walls with pulsatile flowing were developed in [9, and this study contribut- 
ed to simulate hemodynamics. Authors in [10] considered pipes attached by viscoelastic materials as damping for declining vibrations. Furthermore, the dynamic analysis of the viscoelastic pipes is a critical subject for pipes conveying fluid due to the problem of modelling the viscoelastic constitution relation accurately.

In the past decades, the viscoelastic constitution model has been constructed by using the integer order differential operator [11 13. As the fractional order differential operator developing rapidly, the fractional order model is used to the viscoelastic constitution relationship. Comparison to the integer order differential operator, the fractional can always describe the memory property of the viscoelastic material better. Peng et al. [14 deduced a wellbore shrikage model by applying fractional calculus, and verified that fractional viscoelastic constitutive model could fit highly nonlinear behavior. Afterwards, the fractional order model began to be applied on the dynamics of the viscoelastic field. Long et al. 15] proposed four classes of fractional order viscoelastic constitution models, and results showed they can describe the viscoelastic behavior better. Mokhtari et al. [16] applied the fractional Zenner constitution model on the sandwich circular cylindrical shell. Numerical results demonstrated the effect of geometrical parameters on the flutter. The researchers in [17] focused on utilizing fractional operators to accurately describe viscoelastic properties and more complicated dynamic behavior.

It is pointed that the fractional order model is not suitable for representing the behavior of dynamic viscoelastic properties in most cases. In the dynamic analysis, subtle changes in parameters can cause huge changes in the system. The variable fractional order differential operator can better model the viscoelastic constitution relation than the constant fractional order differential operator in large strain [18]. Li et al. 19] studied the shape-memory polymer which had been developed in critical applications due to the memory property. Meng et al. [20] adopted a newly developed method for variable fractional order calculus as a efficient in predicting the compression deformation of amorphous glassy polymers and improving the accuracy of constitutive models with fewer 
parameters.

Whereas there was almost no researches on studying the variable fractional order model on viscoelastic pipes. To increase the accuracy and efficiency of the dynamic analysis, not only the variable fractional order model is treated as the constitution model of viscoelastic pipes conveying fluid, but also a reliable technique is needed to solve the variable fractional equations accurately. AlMdallal and Syam 21] used the Chebyshev collocation-path following method to solve sixth-order Sturm-Liouville problems. More creatively, the authors used the fractional-order Legendre-collocation method [22] and fractional-Legendre spectral Galerkin method 23 to solve fractional initial value problems and fractional Sturm-Liouville problems. Heydari et al. 24] proposed a numerical method to solve linear multi-term variable fractional diffusion-wave equation by the Chebyshev wavelet. Bernstein polynomials method was presented to solve a class variable fractional order linear cable equation [25] and variable order time fractional diffusion equation [26] numerically. The study in [27] aimed at numerically resolving one kind of nonlinear variable fractional differential equations (FDEs) by means of Legendre wavelet approach and operational matrices. Chen et al. 28] used polynomials method to study one kind of nonlinear variable FDEs based on Bernstein polynomials. For integral equations, Assari and Dehghan [29] studied Volterra integral equations by the meshless local Galerkin method. There is few researches about numerical schemes of variable fractional integral-differential equations. And the authors in 30 researched one kind of linear variable fractional order integral-differential equation numerically by Bernstein polynomials method. Moghaddam and Machado [31] investigated a numerical algorithm of a type of variable fractional integral-differential equation$\mathrm{s}$ in view of weakly singular kernels. However, there was almost no researches on studying nonlinear variable fractional order integral-differential equations for numerical solutions.

Based on the above reasons, a variable fractional model and a new method are needed to be applied on this class of problems to improve the accuracy numerical solutions in this paper. For numerically solving this problem, an al- 
gorithm is proposed in this study. The proposed algorithm is not only applied on solving the governing equation with more accurate variable fractional order model, but also used to achieve the dynamic analysis of viscoelastic pipes conveying fluid as a high precise technique. More importantly, Shifted Legendre Polynomials algorithm will increase reliability on predicting viscoelastic behaviors and dynamic properties of pipes conveying fluid problem.

This article aims to study the nonlinear dynamic analysis for viscoelastic pipes conveying fluid in the presence of the external load excitation. The variable fractional order model is chosen as the constitution model to set up pipes dynamic equation in next section. Moreover, its form is the nonlinear differential and integral equation with variable fractional order. Section 3 presented a Shifted Legendre Polynomials algorithm to get numerical solutions of the pipes dynamic equation. Section 4 considered the influences of a varity of parameters on the dynamics of pipes. Finally, conclusions are contained in Section 5.

\section{Mathematical model}

In this section, the mathematical definition and property of variable fractional calculus, a variable fractional order model and the form of a governing equation are introduced. Afterwards, the mentioned definition and property will be used later.

Definition 1 The Caputo fractional derivative operator $D^{\alpha(t)}$ of order $\alpha(t)$ is defined as [27; 32]

$$
D^{\alpha(t)} f(t)=\frac{1}{\Gamma(1-\alpha(t))} \int_{0^{+}}^{t}(t-\tau)^{-\alpha(t)} f^{\prime}(\tau) d \tau
$$

where $t \geq 0,0<\alpha(t) \leq 1, \alpha(t)$ is variable fractional order, $f(t)$ is continuous over interval $(0,+\infty)$ and is integrable over any subinterval of $[0,+\infty)$. 
Based on Definition 1, the variable fractional derivative of a polynomial is represented as

$$
D_{t}^{\alpha(t)} t^{m}= \begin{cases}\frac{\Gamma(m+1)}{\Gamma(m+1-\alpha(t))} t^{m-\alpha(t)}, & m=1,2, \cdots \\ 0, & m=0 .\end{cases}
$$

This property will be available for the algorithm proposed later.

Viscoelastic pipes conveying fluid are considered in this article. The viscoelastic pipe dynamic equation [33] is written as

$$
\rho_{p} A_{p} \frac{\partial^{2} w}{\partial t^{2}}+\rho_{f} A_{f}\left(\frac{\partial^{2} w}{\partial t^{2}}+2 V \frac{\partial^{2} w}{\partial x \partial t}+V^{2} \frac{\partial^{2} w}{\partial x^{2}}\right)-P_{0} \frac{\partial^{2} w}{\partial x^{2}}-\frac{1}{H} \frac{\partial^{2} w}{\partial x^{2}} \int_{0}^{H} A_{p} \sigma d x=f(x, t) .
$$

The boundary conditions are

$$
\frac{\partial w(0, t)}{\partial x}=\frac{\partial w(H, t)}{\partial x}=w(0, t)=w(H, t)=0 .
$$

The variable fractional order model is applied to accurately describe the constitutive relationship of viscoelastic pipes. The constitutive model [18 and strain [33] are respectively formulated as

$$
\begin{gathered}
\sigma(x, t)=E \theta^{\alpha(t)} D_{t}^{\alpha(t)} \varepsilon(x, t) \\
\varepsilon(x, t)=\frac{1}{2}\left(\frac{\partial w}{\partial x}\right)^{2}
\end{gathered}
$$

where $\sigma(x, t)$ is stress, $\varepsilon(x, t)$ is strain, $E, \theta$ and $\alpha(t)$ are the parameters of the viscoelastic constitution model. Also $\alpha(t)$ need to satisfy $0<\alpha(t)<1$.

In this research, it is assumed that viscoelastic pipes have been in a large deformation state after undergoing small deformation and $\alpha(t)$ satisfies $0<$ $\alpha(t)<1$ within the considered time domain. After deriving the formula (3), (5) and (6), the governing equation is expressed as

$$
\begin{gathered}
\left(\rho_{p} A_{p}+\rho_{f} A_{f}\right) \frac{\partial^{2} w}{\partial t^{2}}+2 V \rho_{f} A_{f} \frac{\partial^{2} w}{\partial x \partial t}+\left(V^{2} \rho_{f} A_{f}-P_{0}\right) \frac{\partial^{2} w}{\partial x^{2}} \\
+\frac{E A_{p}}{2 H} \theta^{\alpha(t)} \frac{\partial^{2} w}{\partial x^{2}} \int_{0}^{H} D_{t}^{\alpha(t)}\left(\frac{\partial w}{\partial x}\right)^{2} d x=f(x, t)
\end{gathered}
$$


where $\rho_{p}$ and $A_{p}$ mean the density and the cross-sectional areas of viscoelastic pipes respectively. Similarly, $\rho_{f}$ and $A_{f}$ indicate those of fluid. $f(x, t)$ is the external force excitation. $V, H$ and $P_{0}$ are respectively the fluid velocity, the length of pipes and force at ends of pipes which are constants. $w(x, t)$ is the displacement, $x$ and $t$ are position and time variables. Furthermore, the geometrical relation of pipes is shown in Figure 1

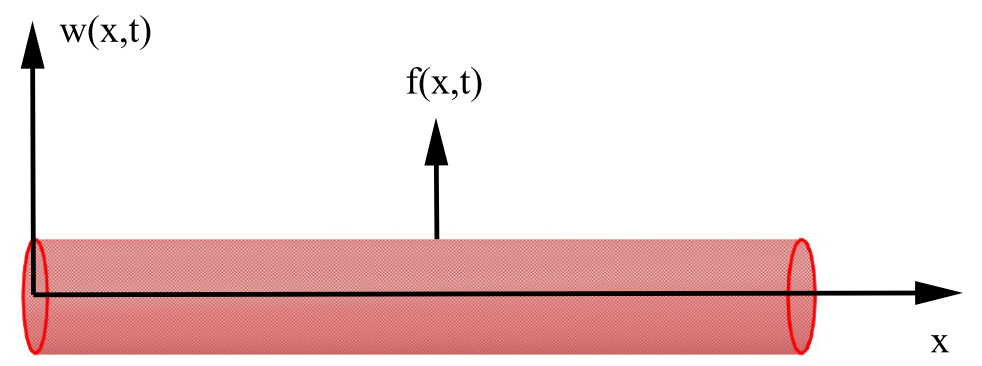

Figure 1: The geometric relation of viscoelastic pipes conveying fluid.

\section{Shifted Legendre Polynomials algorithm}

In this section, Shifted Legendre Polynomials algorithm is illustrated in detail. The Legendre polynomial is a kind of orthogonal polynomials which are used to be the basic function to approximate the analytical function. Compared with other orthogonal polynomials, such as the Chebyshev polynomial [36], the Bernoulli polynomial [37, Hermite polynomial [38 and so on, the Legendre polynomial has a simpler weight function. Especially in the process of approximating nonlinear integral terms, it avoids a mount of calculations due to the simple power function that values 1 . However, its definition interval is $[-1,1]$. In order to better approximate the unknown analytical function in the large interval, the shifted Legendre polynomial is selected in the article. Its key idea is to use shifted Legendre polynomials as the basic function to approximate the unknown analytical function precisely and show derivative function by the term 
of matrix. The governing equation is transformed into the algebraic equation. The parameters of the approximated function are obtained after discreting and solving the algebraic equation. Then numerical solutions will be obtained from the precisely determinate approximated function. Furthermore, the numerical solution is infinitely closed to the exact solution.

\subsection{Shifted Legendre Polynomials}

The shifted Legendre polynomial of degree $n$ in [34, 35] is defined by

$$
l_{n, i}(x)=\sum_{i=0}^{n}(-1)^{n+i} \frac{\Gamma(n+i+1)}{\Gamma(n-i+1)(\Gamma(i+1))^{2}} x^{i}
$$

where $i=0,1, \cdots, n, x \in[0,1]$. Then a matrix $\varphi_{n}(x)$ consisted of a sequence of shifted Legendre polynomials defined in $[0,1]$ is formulated as

$$
\varphi_{n}(x)=\left[l_{n, 0}(x), l_{n, 1}(x), \cdots, l_{n, n}(x)\right]^{T}=A T_{n}(x)
$$

where $T_{n}(x)=\left[1, x, \cdots, x^{n}\right]^{T}$,

$$
A=\left[a_{i j}\right]_{i, j=0}^{n}, \quad a_{i j}= \begin{cases}0, & i<j \\ (-1)^{i+j} \frac{\Gamma(i+j+1)}{\Gamma(i-j+1)(\Gamma(j+1))^{2}}, & i \geq j\end{cases}
$$

$A$ is a $n+1$ order lower triangular matrix and the elements of matrix $A$ on the diagonal are all positive numbers. Matrix $A$ can be transformed into the $n+1$ order identity matrix by the linear transformation. So, matrix $A$ is invertible.

The definition domain of $w(x, t)$, which is used to express the displacement of the pipe in the governing equation $(7)$, is selected as $[0, H] \times[0, S]$. In theory, the definition interval of $t$ is $[0,+\infty]$. However, the viscoelastic pipe conveying fluid can't perform forever. For reducing computations and improving efficiency in practical problems, the interval of $t$ is selected as a limited interval $[0, S]$ where $S$ values an arbitrary positive number based on needs.

In order to expand the range of $x$, the shifted Legendre polynomial of degree 
$n$ in $[0, H]$ can be written

$$
\begin{aligned}
L_{n, i}(x) & =\sum_{i=0}^{n}(-1)^{n+i} \frac{\Gamma(n+i+1)}{\Gamma(n-i+1)(\Gamma(i+1))^{2}}\left(\frac{x}{H}\right)^{i} \\
& =\sum_{i=0}^{n}(-1)^{n+i} \frac{\Gamma(n+i+1)}{\Gamma(n-i+1)(\Gamma(i+1))^{2}}\left(\frac{1}{H}\right)^{i} x^{i}
\end{aligned}
$$

where $i=0,1, \cdots, n, x \in[0, H]$.

Then a matrix $\Phi_{n}(x)$ composed of a sequence of shifted Legendre polynomials defined in $[0, H]$ is formulated as

$$
\Phi_{n}(x)=\left[L_{n, 0}(x), L_{n, 1}(x), \cdots, L_{n, n}(x)\right]^{T}=A L T_{n}(x)
$$

where

$$
L=\left[l_{i j}\right]_{i, j=0}^{n}, \quad l_{i j}= \begin{cases}0, & i \neq j \\ H^{-i}, & i=j .\end{cases}
$$

$L$ is a $n+1$ order diagonal matrix and the elements of matrix $L$ on the diagonal are all positive numbers. Then $L$ is invertible.

The shifted Legendre polynomial of degree $n$ in $[0, S]$ is expressed as

$$
\begin{aligned}
\bar{L}_{n, i}(t) & =\sum_{i=0}^{n}(-1)^{n+i} \frac{\Gamma(n+i+1)}{\Gamma(n-i+1)(\Gamma(i+1))^{2}}\left(\frac{t}{S}\right)^{i} \\
& =\sum_{i=0}^{n}(-1)^{n+i} \frac{\Gamma(n+i+1)}{\Gamma(n-i+1)(\Gamma(i+1))^{2}}\left(\frac{1}{S}\right)^{i} t^{i}
\end{aligned}
$$

where $i=0,1, \cdots, n, t \in[0, S]$.

Then a matrix $\phi_{n}(t)$ composed of a series of shifted Legendre polynomials defined in $[0, S]$ is expressed as

$$
\phi_{n}(t)=\left[\bar{L}_{n, 0}(t), \bar{L}_{n, 1}(t), \cdots, \bar{L}_{n, n}(t)\right]^{T}=A M T_{n}(t)
$$

where

$$
M=\left[m_{i j}\right]_{i, j=0}^{n}, \quad m_{i j}= \begin{cases}0, & i \neq j \\ S^{-i}, & i=j .\end{cases}
$$

$M$ is a $n+1$ order diagonal matrix and the elements of $M$ on the diagonal are all positive numbers. Then $M$ is invertible. 


\subsection{Function approximation}

A continuous function $w(x)$ in the domain $[0, H]$ can be expanded in terms of shifted Legendre polynomials as $w(x)=\lim _{n \rightarrow \infty} \sum_{i=0}^{n} c_{i} L_{n, i}(x), w(x)$ can be approximated as

$$
w(x) \approx w_{n}(x)=\sum_{i=0}^{n} c_{i} L_{n, i}(x)=C^{T} \Phi_{n}(x)
$$

where $C^{T}=\left[c_{0}, c_{1}, \cdots, c_{n}\right]$. Then

$$
C^{T}\left\langle\Phi_{n}(x), \Phi_{n}^{T}(x)\right\rangle=\left\langle w(x), \Phi_{n}^{T}(x)\right\rangle .
$$

Let

$$
Q=\left\langle\Phi_{n}(x), \Phi_{n}^{T}(x)\right\rangle=\left[\delta_{i j}\right]_{i, j=0}^{n}
$$

where $\delta_{i j}=\int_{0}^{H} L_{n, i}(x) L_{n, j}(x) d x=\left\{\begin{array}{ll}0, & i \neq j \\ \frac{H}{i+j+1}, & i=j\end{array}(i, j=0,1, \cdots, n)\right.$.

$Q$ is a $n+1$ order diagonal matrix and the elements of $Q$ on the diagonal are all positive numbers. Then $Q$ is invertible. So,

$$
C^{T}=\left\langle w(x), \Phi_{n}^{T}(x)\right\rangle Q^{-1}
$$

Similarly, a continuous function $w(t)$ in the domain $[0, S]$ can be expanded in terms of shifted Legendre polynomials as $w(t)=\lim _{n \rightarrow \infty} \sum_{i=0}^{n} k_{i} \bar{L}_{n, i}(t), w(t)$ is represented by

$$
w(t) \approx w_{n}(t)=\sum_{i=0}^{n} k_{i} \bar{L}_{n, i}(t)=K^{T} \phi_{n}(t)
$$

where $K^{T}=\left[k_{0}, k_{1}, \cdots, k_{n}\right]$. Then

$$
K^{T}\left\langle\Phi_{n}(t), \Phi_{n}^{T}(t)\right\rangle=\left\langle w(t), \Phi_{n}^{T}(t)\right\rangle .
$$

Let

$$
P=\left\langle\Phi_{n}(t), \Phi_{n}^{T}(t)\right\rangle=\left[\Delta_{i j}\right]_{i, j=0}^{n}
$$

where $\Delta_{i j}=\int_{0}^{S} \bar{L}_{n, i}(t) \bar{L}_{n, j}(t) d t=\left\{\begin{array}{ll}0, & i \neq j \\ \frac{S}{i+j+1}, & i=j\end{array}(i, j=0,1, \cdots, n)\right.$.

$P$ is a $n+1$ order diagonal matrix and the elements of $P$ on the diagonal are 
all positive numbers. Then $P$ is invertible. So,

$$
K^{T}=\left\langle w(t), \Phi_{n}^{T}(t)\right\rangle P^{-1} .
$$

Two-variable continuous function $w(x, t)$ defined on $\Lambda=[0, H] \times[0, S]$ may be written as

$$
\begin{aligned}
w(x, t) & =\lim _{n \rightarrow \infty} \sum_{j=0}^{n}\left(\sum_{i=0}^{n} c_{i} L_{n, i}(x)\right) k_{j} \bar{L}_{n, j}(t) \\
& =\lim _{n \rightarrow \infty} \sum_{j=0}^{n} \sum_{i=0}^{n} c_{i} k_{j} L_{n, i}(x) \bar{L}_{n, j}(t) \\
& =\lim _{n \rightarrow \infty} \sum_{j=0}^{n} \sum_{i=0}^{n} w_{i j} L_{n, i}(x) \bar{L}_{n, j}(t) \\
& =\lim _{n \rightarrow \infty} \Phi_{n}(x) U \phi_{n}(t)
\end{aligned}
$$

where $U=\left[w_{i j}\right]_{i, j=0}^{n}$ and $w_{i j}=c_{i} k_{j}$.

\subsection{Differential operator matrix}

$A, L$ and $M$ are $n+1$ order invertible matrices proven in the above parts. So, matrix $A L$ and $A M$, which will be used in this part, are also invertible.

$\Phi_{n}(x)$ is a series of polynomial matrices with respect to $x$, the derivative of $\Phi_{n}(x)$ with respect to $x$ is formulated as

$$
\frac{d \Phi_{n}(x)}{d x}=D_{x} \Phi_{n}(x) .
$$

Then

$$
D_{x} \Phi_{n}(x)=D_{x} A L T_{n}(x)=A L \frac{d T_{n}(x)}{d x}=A L E T_{n}(x)=A L E(A L)^{-1} \Phi_{n}(x)
$$

where $E=\left[e_{i j}\right]_{i, j=0}^{n}, \quad e_{i j}=\left\{\begin{array}{cc}0, & i \neq j+1 \\ i, & i=j+1\end{array}\right.$.

By virtual of (24), differential operator matrix $D_{x}$ is obtained as

$$
D_{x}=A L E^{m}(A L)^{-1} .
$$

The $m$ exponent th derivative of $\Phi_{n}(x)$ with respect to $x$ can be obtained

$$
\frac{d^{m} \Phi_{n}(x)}{d x^{m}}=D_{m x} \Phi_{n}(x), \quad m \in N
$$


Then

$$
\begin{aligned}
D_{m x} \Phi_{n}(x) & =D_{m x} A L T_{n}(x)=A L \frac{d^{m} T_{n}(x)}{d x^{m}} \\
& =A L E^{m} T_{n}(x)=A L E^{m}(A L)^{-1} \Phi_{n}(x) .
\end{aligned}
$$

Based on formula (27), differential operator matrix $D_{m x}$ can be obtained as

$$
D_{m x}=A L E^{m}(A L)^{-1} .
$$

Similarly, $\phi_{n}(t)$ is a series of polynomial matrices with respect to $t$, the derivative of $\phi_{n}(t)$ with respect to $t$ can be gotten

$$
\frac{d \phi_{n}(t)}{d t}=D_{t} \phi_{n}(t) .
$$

Then

$$
\begin{aligned}
D_{t} \phi_{n}(t) & =D_{t} A M T_{n}(t)=A M \frac{d T_{n}(t)}{d t} \\
& =A M E T_{n}(t)=A M E(A M)^{-1} \phi_{n}(t) .
\end{aligned}
$$

Therefore, from (30), differential operator matrix $D_{t}$ is obtained as

$$
D_{t}=A M E(A M)^{-1} .
$$

The $v$ exponent th derivative of $\phi_{n}(t)$ with respect to $t$ is written by

$$
\frac{d^{v} \phi_{n}(t)}{d t^{v}}=D_{v t} \phi_{n}(t), \quad v \in N .
$$

Then

$$
\begin{aligned}
D_{v t} \phi_{n}(t) & =D_{v t} A M T_{n}(t)=A M \frac{d^{v} T_{n}(t)}{d t^{v}} \\
& =A M E^{v} T_{n}(t)=A M E^{v}(A M)^{-1} \phi_{n}(t) .
\end{aligned}
$$

From formula (33), differential operator matrix $D_{v t}$ is obtained as

$$
D_{v t}=A M E^{v}(A M)^{-1} .
$$

Thus,

$$
\frac{\partial^{m+v} u(x, t)}{\partial x^{m} \partial t^{v}} \approx\left(D_{m x} \Phi_{n}(x)\right)^{T} U\left(D_{v t} \phi_{n}(t)\right)
$$

where only $U$ is unknown. 
3.4. Processing of the nonlinear integral-differential term $w(x, t)$ is approximately shown as

$$
w(x, t) \approx w_{n}(x, t)=(A L X)^{T} U(A M T)
$$

where $X=T_{n}(x), T=T_{n}(t)$. Thus,

$$
\begin{aligned}
& \left(\frac{\partial w(x, t)}{\partial x}\right)^{2} \approx\left(\frac{\partial w_{n}(x, t)}{\partial x}\right)^{2}=\left(\frac{\partial w_{n}(x, t)}{\partial x}\right)^{T}\left(\frac{\partial w_{n}(x, t)}{\partial x}\right) \\
= & (A M T)^{T} U^{T} D_{x}(A L X)(A L X)^{T} D_{x}^{T} U(A M T) .
\end{aligned}
$$

The integration over the interval $[0, H]$ of $w^{2}(x, t)$ can be denoted as

$$
\begin{array}{rl}
\int_{0}^{H}\left(\frac{\partial w(x, t)}{\partial x}\right)^{2} & d x \approx \int_{0}^{H}(A M T)^{T} U^{T} D_{x}(A L X)(A L X)^{T} D_{x}^{T} U(A M T) d x \\
= & (A M T)^{T} U^{T} D_{x} \int_{0}^{H}(A L X)(A L X)^{T} d x D_{x}^{T} U(A M T) \\
= & (A M T)^{T} U^{T} D_{x} Q D_{x}^{T} U(A M T) .
\end{array}
$$

Therefore, the nonlinear integral-differential term with variable fractional order is demonstrated as

$$
\begin{aligned}
& \int_{0}^{H} D_{t}^{\alpha(t)}\left(\frac{\partial w(x, t)}{\partial x}\right)^{2} d x \\
\approx & \int_{0}^{H} D_{t}^{\alpha(t)}\left[(A M T)^{T} U^{T} D_{x}(A L X)(A L X)^{T} D_{x}^{T} U(A M T)\right] d x \\
= & \int_{0}^{H} D_{t}^{\alpha(t)}\left(T^{T} G T\right) d x \\
= & \int_{0}^{H} T^{T} R T d x \\
= & T^{T} B T
\end{aligned}
$$

where $G=(A M)^{T} U^{T} D_{x}(A L X)(A L X)^{T} D_{x}^{T} U(A M)=\left[g_{i j}\right]_{i, j=0}^{n}, \quad R=\left[r_{i j}\right]_{i, j=0}^{n}$,

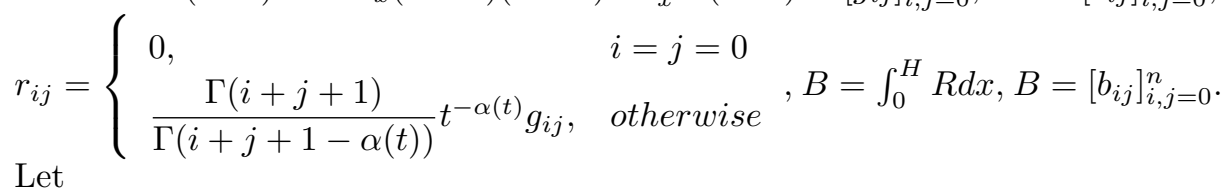

$$
\begin{aligned}
F & =\int_{0}^{H} G d x=(A M)^{T} U^{T} D_{x} \int_{0}^{H}(A L X)(A L X)^{T} d x D_{x}^{T} U(A M) \\
& =(A M)^{T} U^{T} D_{x} Q D_{x}^{T} U(A M)
\end{aligned}
$$


where $F=\left[f_{i j}\right]_{i, j=0}^{n}, f_{i j}=\int_{0}^{H} g_{i j} d x$. Then

$$
\begin{aligned}
b_{i j} & =\int_{0}^{H} r_{i j} d x \\
& = \begin{cases}0, & i=j=0 \\
\frac{\Gamma(i+j+1)}{\Gamma(i+j+1-\alpha(t))} t^{-\alpha(t)} \int_{0}^{H} g_{i j} d x, & \text { otherwise }\end{cases} \\
& = \begin{cases}0, & i=j=0 \\
\frac{\Gamma(i+j+1)}{\Gamma(i+j+1-\alpha(t))} t^{-\alpha(t)} f_{i j}, & \text { otherwise. }\end{cases}
\end{aligned}
$$

Similarly, the known function $f(x, t)$ can also be expressed as the following form:

$$
f(x, t) \approx \Phi_{n}^{T}(x) U^{*} \phi_{n}(t)
$$

where $U^{*}$ is a known $n+1$ order matrix after solving by the shifted Legendre polynomials.

Finally, the governing equation (7) is transformed into a algebraic equation with unknown $U$ :

$$
\begin{aligned}
& \left(\rho_{p} A_{p}+\rho_{f} A_{f}\right) \Phi_{n}^{T}(x) U\left(D_{2 t} \phi_{n}(t)\right)+2 V \rho_{f} A_{f}\left(D_{x} \Phi_{n}(x)\right)^{T} U\left(D_{t} \phi_{n}(t)\right) \\
+ & \left(V^{2} \rho_{f} A_{f}-P_{0}\right)\left(D_{2 x} \Phi_{n}(x)\right)^{T} U \phi_{n}(t)+\frac{E A_{p}}{2 H} \theta^{\alpha(t)}\left(D_{2 x} \Phi_{n}(x)\right)^{T} U \phi_{n}(t) T^{T} B T=\Phi_{n}^{T}(x) U^{*} \phi_{n}(t) .
\end{aligned}
$$

To discretize $x$ and $t$ as points $\left(x_{i}, t_{j}\right)$, where $x_{i}=i \frac{H}{n}$ and $t_{j}=j \frac{S}{n}, U$ can be obtained based on the least square method. Numerical solutions $w_{n}(x, t)$ will be obtained. Also, $w_{n}(x, t)$ can be seen as the interpolating polynomial of $w(x, t)$ at points $\left(x_{i}, t_{j}\right)$.

\subsection{Convergence analysis and error bounds}

The aim of this part is to study the convergence and error bound of the proposed numerical algorithm. The convergence and the estimate of the error bound for the approximate solution of the governing equation (7) are investigated on $\Lambda=[0, H] \times[0, S]$. For doing this, the Banach space $W=C[0, H] \times C[0, S]$ 
is considered with the following norm:

$$
\|w(x, t)\|=\langle w(x, t), w(x, t)\rangle^{\frac{1}{2}}=\left(\int_{0}^{S} \int_{0}^{H}|w(x, t)|^{2} d x d t\right)^{\frac{1}{2}} .
$$

Theorem 1 Suppose that $w(x, t)$ and $w_{n}(x, t)$ are the exact solution and approximate solution obtained by the Shifted Legendre Polynomials algorithm in the Banach space $W=C[0, H] \times C[0, S]$. Then

$$
\begin{aligned}
\left\|w_{n}(x, t)-w(x, t)\right\| \leq & (H S)^{\frac{1}{2}}\left(\frac{1}{4} A_{1}\left(\frac{H}{n}\right)^{n+1}+\frac{1}{4} A_{2}\left(\frac{S}{n}\right)^{n+1}\right. \\
& \left.+\frac{1}{16} A_{3}\left(\frac{H}{n}\right)^{n+1}\left(\frac{S}{n}\right)^{n+1}\right)
\end{aligned}
$$

where $A_{1}, A_{2}$ and $A_{3}$ are terminate constants.

Proof $w_{n}(x, t)$ is the numerical solution obtained as the interpolating polynomial of $w(x, t)$ at points $\left(x_{i}, t_{j}\right)$ by means of Shifted Legendre Polynomials algorithm, where $x_{i}=i \frac{H}{n}$ and $t_{j}=j \frac{S}{n}$. Then, one can obtain

$$
\begin{aligned}
w(x, t)-w_{n}(x, t)= & \frac{\partial^{n+1} w(\mu, t)}{\partial x^{n+1}} \frac{\prod_{i=0}^{n}\left(x-x_{i}\right)}{(n+1) !}+\frac{\partial^{n+1} w(x, \xi)}{\partial t^{n+1}} \frac{\prod_{j=0}^{n}\left(t-t_{j}\right)}{(n+1) !} \\
& -\frac{\partial^{2 n+2} w\left(\mu^{\prime}, \xi^{\prime}\right)}{\partial x^{n+1} \partial t^{n+1}} \frac{\prod_{i=0}^{n}\left(x-x_{i}\right) \prod_{j=0}^{n}\left(t-t_{j}\right)}{[(n+1) !]^{2}}
\end{aligned}
$$

for $\mu, \mu^{\prime} \in[0, H]$ and $\xi, \xi^{\prime} \in[0, S]$. Thus, one can have

$$
\begin{aligned}
\left|w_{n}(x, t)-w(x, t)\right| \leq & \max _{(x, t) \in \bigwedge}\left|\frac{\partial^{n+1} w(x, t)}{\partial x^{n+1}}\right| \frac{\prod_{i=0}^{n}\left|x-x_{i}\right|}{(n+1) !} \\
& +\max _{(x, t) \in \bigwedge}\left|\frac{\partial^{n+1} w(x, t)}{\partial t^{n+1}}\right| \frac{\prod_{j=0}^{n}\left|t-t_{j}\right|}{(n+1) !} \\
& +\max _{(x, t) \in \bigwedge}\left|\frac{\partial^{2 n+2} w(x, t)}{\partial x^{n+1} \partial t^{n+1}}\right| \frac{\prod_{i=0}^{n}\left|x-x_{i}\right| \prod_{j=0}^{n}\left|t-t_{j}\right|}{[(n+1) !]^{2}} .
\end{aligned}
$$


To find the bounds on $\prod_{i=0}^{n}\left|x-x_{i}\right|$ and $\prod_{j=0}^{n}\left|t-t_{j}\right|$, defining the variables $x=\eta \frac{H}{n}$ and $t=\eta^{\prime} \frac{S}{n}$, it gives that

$$
\begin{aligned}
& \prod_{i=0}^{n}\left|x-x_{i}\right|=\left(\frac{H}{n}\right)^{n+1} \prod_{i=0}^{n}|\eta-i| \\
& \prod_{j=0}^{n}\left|t-t_{j}\right|=\left(\frac{S}{n}\right)^{n+1} \prod_{j=0}^{n}\left|\eta^{\prime}-j\right| .
\end{aligned}
$$

By choosing the integers $n_{1}$ and $n_{2}$ which are less than $n$, we have $\eta \in\left(n_{1}, n_{1}+1\right)$ and $\eta^{\prime} \in\left(n_{2}, n_{2}+1\right)$, therefore

$$
\begin{aligned}
& \prod_{i=0}^{n}|\eta-i|=\left|\left(\eta-n_{1}\right)\left(\eta-n_{1}-1\right)\right| \prod_{i=0}^{n_{1}-1}|\eta-i| \prod_{i=n_{1}+2}^{n}|\eta-i| \\
& \prod_{j=0}^{n}\left|\eta^{\prime}-j\right|=\left|\left(\eta^{\prime}-n_{2}\right)\left(\eta^{\prime}-n_{2}-1\right)\right| \prod_{i=0}^{n_{2}-1}\left|\eta^{\prime}-j\right| \prod_{j=n_{2}+2}^{n}\left|\eta^{\prime}-j\right| .
\end{aligned}
$$

Note that $\left|\left(\eta-n_{1}\right)\left(\eta-n_{1}-1\right)\right|$ and $\left|\left(\eta^{\prime}-n_{2}\right)\left(\eta^{\prime}-n_{2}-1\right)\right|$ have maximum when $\eta$ and $\eta^{\prime}$ values at points $n_{1}+\frac{1}{2}$ and $n_{2}+\frac{1}{2}$, respectively, thus

$$
\begin{aligned}
& \left|\left(\eta-n_{1}\right)\left(\eta-n_{1}-1\right)\right| \leq \frac{1}{4} \\
& \left|\left(\eta^{\prime}-n_{2}\right)\left(\eta^{\prime}-n_{2}-1\right)\right| \leq \frac{1}{4} .
\end{aligned}
$$

And we can get

$$
\begin{aligned}
\prod_{i=0}^{n_{1}-1}|\eta-i| \prod_{i=n_{1}+2}^{n}|\eta-i| & \leq \prod_{i=0}^{n_{1}-1}\left(n_{1}+1-i\right) \prod_{i=n_{1}+2}^{n}\left(i-n_{1}\right) \\
& =\left(n_{1}+1\right) !\left(n-n_{1}\right) ! \leq(n+1) !
\end{aligned}
$$

and

$$
\begin{aligned}
\prod_{i=0}^{n_{2}-1}\left|\eta^{\prime}-i\right| \prod_{i=n_{2}+2}^{n}\left|\eta^{\prime}-i\right| & \leq \prod_{i=0}^{n_{2}-1}\left(n_{2}+1-i\right) \prod_{i=n_{2}+2}^{n}\left(i-n_{2}\right) \\
& =\left(n_{2}+1\right) !\left(n-n_{2}\right) ! \leq(n+1) !
\end{aligned}
$$

Substituting (50)- $(52)$ into $(49)$, it yields

$$
\begin{aligned}
\prod_{i=0}^{n}|\eta-i| & =\left|\left(\eta-n_{1}\right)\left(\eta-n_{1}-1\right)\right| \prod_{i=0}^{n_{1}-1}|\eta-i| \prod_{i=n_{1}+2}^{n}|\eta-i| \\
& \leq \frac{1}{4}(n+1) !
\end{aligned}
$$


and

$$
\begin{aligned}
\prod_{j=0}^{n}\left|\eta^{\prime}-j\right| & =\left|\left(\eta^{\prime}-n_{2}\right)\left(\eta^{\prime}-n_{2}-1\right)\right| \prod_{j=0}^{n_{2}-1}\left|\eta^{\prime}-j\right| \prod_{j=n_{2}+2}^{n}\left|\eta^{\prime}-j\right| \\
& \leq \frac{1}{4}(n+1) ! .
\end{aligned}
$$

Therefore, according to (48), (53) and (54), one has

$$
\begin{aligned}
& \prod_{i=0}^{n}\left|x-x_{i}\right| \leq\left(\frac{H}{n}\right)^{n+1} \frac{1}{4}(n+1) ! \\
& \prod_{j=0}^{n}\left|t-t_{j}\right| \leq\left(\frac{S}{n}\right)^{n+1} \frac{1}{4}(n+1) ! .
\end{aligned}
$$

Considering $A_{1}=\max _{(x, t) \in \Lambda}\left|\frac{\partial^{n+1} w(x, t)}{\partial x^{n+1}}\right|, A_{2}=\max _{(x, t) \in \Lambda}\left|\frac{\partial^{n+1} w(x, t)}{\partial t^{n+1}}\right|$ and $A_{3}=\max _{(x, t) \in \Lambda}\left|\frac{\partial^{2 n+2} w(x, t)}{\partial x^{n+1} \partial t^{n+1}}\right|$, gives

$$
\left|w_{n}(x, t)-w(x, t)\right| \leq \frac{1}{4} A_{1}\left(\frac{H}{n}\right)^{n+1}+\frac{1}{4} A_{2}\left(\frac{S}{n}\right)^{n+1}+\frac{1}{16} A_{3}\left(\frac{H}{n}\right)^{n+1}\left(\frac{S}{n}\right)^{n+1} .
$$

So,

$$
\begin{aligned}
\left\|w_{n}(x, t)-w(x, t)\right\| \leq & (H S)^{\frac{1}{2}}\left(\frac{1}{4} A_{1}\left(\frac{H}{n}\right)^{n+1}+\frac{1}{4} A_{2}\left(\frac{S}{n}\right)^{n+1}\right. \\
& \left.+\frac{1}{16} A_{3}\left(\frac{H}{n}\right)^{n+1}\left(\frac{S}{n}\right)^{n+1}\right) .
\end{aligned}
$$

The proof of Theorem 1 is completed.

According to (56) and (57), the error bound of the absolute value form is $\left(\frac{1}{4} A_{1}\left(\frac{H}{n}\right)^{n+1}+\frac{1}{4} A_{2}\left(\frac{S}{n}\right)^{n+1}+\frac{1}{16} A_{3}\left(\frac{H}{n}\right)^{n+1}\left(\frac{S}{n}\right)^{n+1}\right)$ and the error bound of the space $L^{2}(\bigwedge)$ norm form is $(H S)^{\frac{1}{2}}\left(\frac{1}{4} A_{1}\left(\frac{H}{n}\right)^{n+1}+\frac{1}{4} A_{2}\left(\frac{S}{n}\right)^{n+1}+\frac{1}{16} A_{3}\left(\frac{H}{n}\right)^{n+1}\left(\frac{S}{n}\right)^{n+1}\right)$. In other words, $\left|w_{n}(x, t)-w(x, t)\right| \rightarrow 0, \forall(x, t) \in \Lambda$ as $n \rightarrow \infty$. Also, $\left\|w_{n}(x, t)-w(x, t)\right\| \rightarrow 0, \forall(x, t) \in \bigwedge$ as $n \rightarrow \infty$.

\subsection{Numerical example}

Consider the following numerical example which is similar to the type of the governing equation(7)

$$
\begin{aligned}
& 5 \frac{\partial^{2} w(x, t)}{\partial t^{2}}+10 \frac{\partial^{2} w(x, t)}{\partial x \partial t}+20 \frac{\partial^{2} w(x, t)}{\partial x^{2}} \\
- & \frac{0.01^{\alpha(t)}}{(10+\sin t)^{2}} \frac{\partial^{2} w(x, t)}{\partial x^{2}} \int_{0}^{L} D_{t}^{\alpha(t)}\left(\frac{\partial w(x, t)}{\partial x}\right)^{2} d x=f(x, t) .
\end{aligned}
$$


The boundary conditions are

$$
\omega(0, t)=0 \quad \omega(0.5, t)=0 \quad \frac{\partial w(0, t)}{\partial x}=0 \quad \frac{\partial w(0.5, t)}{\partial x}=0
$$

where $f(x, t)=10 x^{2}(0.5-x)^{2}+20\left(4 x^{3}-3 x^{2}+0.5 x\right) t+20\left(12 x^{2}-6 x+0.5\right) t^{2}$ $-0.0235 \frac{0.01^{\alpha(t)}}{(10+\sin t)^{2}}\left(12 x^{2}-6 x+0.5\right) t^{6-\alpha(t)}, \alpha(t)=\left\{\begin{array}{ll}0.69, & 0 \leq t<0.5 \\ 0.7-0.02 t, & t \geq 0.5\end{array}\right.$. $\alpha(t)$ can also be written as $\alpha(t)=0.69-|0.005-0.01 t|+0.005-0.01 t$ and the function $f(x, t)$ has been so chosen that the exact solution is $w(x, t)=$ $x^{2}(0.5-x)^{2} t^{2}, x \in[0,0.5], t \in[0,2]$.

Table 1: The absolute error $e_{w}(x, t)$ of the numerical example when $n$ values 6 .

\begin{tabular}{cccccc}
\hline$e_{w}(x, t)$ & $t=0.2$ & $t=0.4$ & $t=0.6$ & $t=0.8$ & $t=1.0$ \\
\hline$x=0.1$ & $0.12 \times 10^{-9}$ & $0.96 \times 10^{-9}$ & $0.34 \times 10^{-9}$ & $2.52 \times 10^{-9}$ & $4.47 \times 10^{-9}$ \\
$x=0.2$ & $0.05 \times 10^{-9}$ & $0.75 \times 10^{-9}$ & $0.34 \times 10^{-9}$ & $2.60 \times 10^{-9}$ & $5.86 \times 10^{-9}$ \\
$x=0.3$ & $0.12 \times 10^{-9}$ & $0.67 \times 10^{-9}$ & $0.19 \times 10^{-9}$ & $1.96 \times 10^{-9}$ & $4.47 \times 10^{-9}$ \\
$x=0.4$ & $0.32 \times 10^{-9}$ & $0.73 \times 10^{-9}$ & $0.05 \times 10^{-9}$ & $0.86 \times 10^{-9}$ & $1.10 \times 10^{-9}$ \\
\hline
\end{tabular}

The variable fractional order nonlinear integral-differential equation example is solved by the proposed algorithm when $n$ is selected as 6 . The numerical solution obtained by the presented algorithm is expressed as $w_{n}(x, t)$. The absolute error $e_{w}(x, t)$ formulated as $e_{w}(x, t)=\left|w_{n}(x, t)-w(x, t)\right|$ of exact and numerical solution is considered in this part for verifying the accuracy of the numerical algorithm. The numerical solution and the exact solution is highly consistent on values in Figure 2(a) The absolute error is shown in Figure 2(b) and it verifies high enough accuracy. However, values of the error seem smaller when $t$ is less than 1 . The specific absolute error is shown in Table 1 and its order of magnitude is less than or equal to $10^{-9}$. 


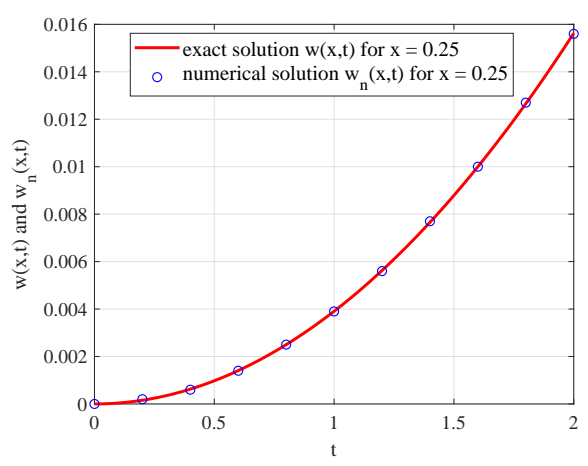

(a) Exact solution and numerical solution

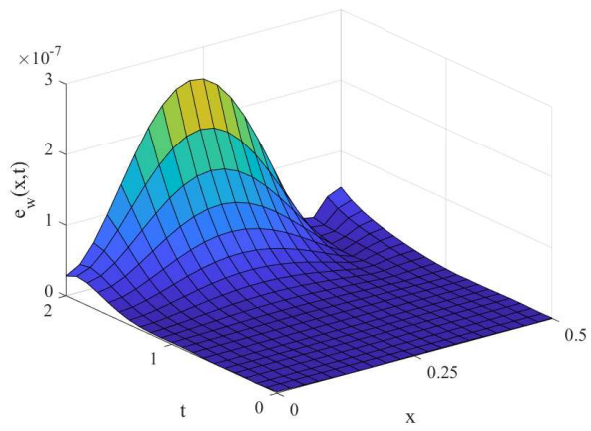

(b) Absolute error

Figure 2: The numerical example when $n$ values 6 for (a) Exact solution and numerical solution and (b) Absolute error.

The numerical example shows that the proposed algorithm has sufficient accuracy to be applied on solving nonlinear variable fractional order integraldifferential equations. The numerical solution $w_{n}(x, t)$ is much close to the exact solution when $n$ is selected as a small value. Also, this algorithm provides an effective technique to analyze viscoelastic pipes conveying fluid numerically. More importantly, the absolute error $e_{w}(x, t)$ has a bound according to Theorem 1 and $e_{w}(x, t) \rightarrow 0, \forall(x, t) \in \bigwedge$ as $n \rightarrow \infty$. In other words, the numerical solution $w_{n}(x, t)$ obtained by the Shifted Legendre Polynomials algorithm is the exact solution $w(x, t)$ when $n$ is increasing to tend to infinity.

\section{Numerical results and dynamic analysis}

The effect of parameters on viscoelastic dynamic behavior and the dynamic properties of pipes are investigated and solved numerically by the Shifted Legendre Polynomials algorithm in this section. The Shifted Legendre Polynomials algorithm has been verified from the convergence analysis and numerical example in mathematical aspect. Several examples are given to not only study the effect of the parameters on viscoelastic pipes, but also check the correctness of 
the numerical computation scheme on specific examples in this section. The variable fractional order model and the governing equation are proposed in this paper for the first time. There are almost no references and methods which can be used to compare with the presented algorithm about viscoelastic pipes conveying fluid under variable fractional order model. So, several examples with other methods [39 41] are considered as the particular example of the study content of this paper to verify the correctness of Shifted Legendre Polynomials algorithm from specific example aspect. It is noted that $n$ is selected as 6 when using the presented numerical algorithm in this section. The displacement $w(x, t)$, velocity $v(x, t)$, acceleration $a(x, t)$, strain $\varepsilon(x, t)$ and stress $\sigma(x, t)$ are considered on dynamic behavior of viscoelastic pipes

$$
\begin{gathered}
v(x, t)=\frac{\partial w(x, t)}{\partial t} \\
a(x, t)=\frac{\partial^{2} w(x, t)}{\partial t^{2}} \\
\sigma(x, t)=\frac{E}{2} \theta^{\alpha(t)} D_{t}^{\alpha(t)}\left(\frac{\partial w(x, t)}{\partial x}\right)^{2} .
\end{gathered}
$$

In this part, these parameters are always considered as $\rho_{p}=1.2 \times 10^{3} \mathrm{~kg} / \mathrm{m}^{3}$, $A_{p}=0.2 \mathrm{~m}^{2}, \rho_{f}=1.05 \times 10^{3} \mathrm{~kg} / \mathrm{m}^{3}, A_{f}=0.05 \mathrm{~m}^{2}$, other parameters are mutative in order to study the effect of changing parameters on pipes. 


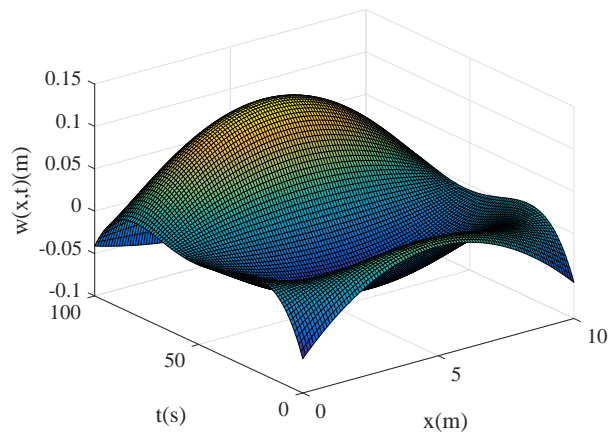

(a) Three-dimensional displacement map

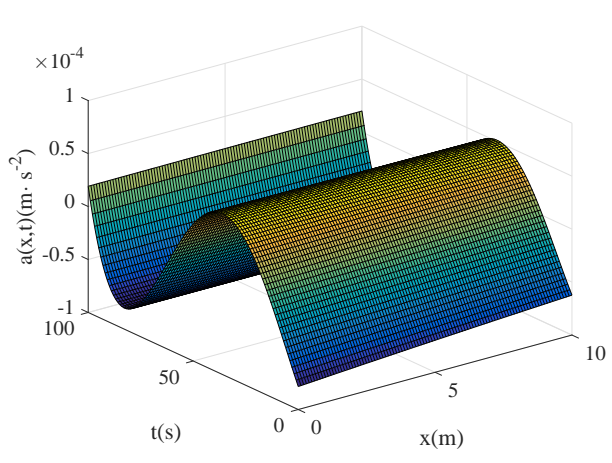

(c) Three-dimensional acceleration map

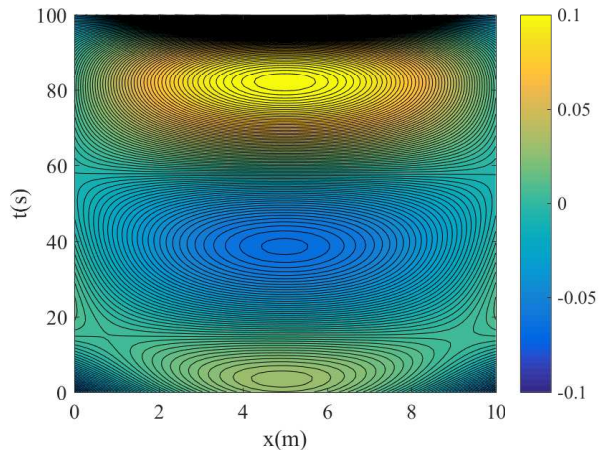

(b) Contour displacement map

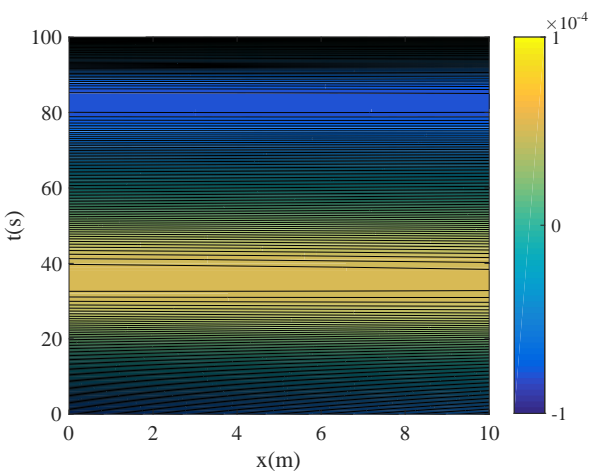

(d) Contour acceleration map

Figure 3: Dynamic response on viscoelastic pipes conveying fluid versus the time and the position when $n$ values 6 for (a) Three-dimensional displacement map, (b) Contour displacement map, (c) Three-dimensional acceleration map and (d) Contour acceleration map. $(f(x, t)=(100+200 \sin (50 t)) \mathrm{N} / \mathrm{m}, E=80 \mathrm{GPa}$, $\theta=0.01, \alpha(t)=0.69-|0.005-0.001 t|+0.005-0.001 t, V=5 \mathrm{~m} / \mathrm{s}, H=10 \mathrm{~m})$

Considering a viscoelastic pipe, its parameters are $f(x, t)=(100+200 \sin (50 t))$ $\mathrm{N} / \mathrm{m}, E=80 \mathrm{GPa}, \theta=0.01, \alpha(t)=0.69-|0.005-0.001 t|+0.005-0.001 t$, $V=5 \mathrm{~m} / \mathrm{s}, H=2 \mathrm{~m}$. In Figure $3(\mathrm{a})$ and Figure 3(c), the displacement $w(x, t)$ and acceleration $a(x, t)$ are changing as wave types. However, its frequency differs too much with force excitation frequency. This denotes the amplitude and 
acceleration response of viscoelastic pipes are not consistent with the excitation response. This is also the specific performance of viscoelastic materials with excellent damping effect. $w(x, t)$ is changing with position $x$ while $a(x, t)$ is almost not in Figure 3(b) and Figure 3(d). Then, it is shown in the following part that how the parameters influence the dynamic behavior of viscoelastic pipes.

\subsection{Dynamic behaviors analysis}

The viscoelastic pipes conveying fluid under $f(x, t)=100 \sin (\omega t) \mathrm{N} / \mathrm{m}, E=$ $80 \mathrm{GPa}, \theta=0.01, \alpha(t)=0.6-|0.005-0.001 t|+0.005-0.001 t, V=5 \mathrm{~m} / \mathrm{s}$, $H=2 \mathrm{~m}$ are considered to study the response of frequency. It can be seen that maximum displacement and acceleration surge when frequency $\omega$ values $111 \mathrm{~Hz}$ and $\alpha_{0}$ values 0.6 in Figure 4(a) and Figure 4(b). While the curves oscillate in a small amplitude when $\omega$ values others over $(100,120)$ and $\alpha_{0}$ values $0.58,0.59$ and 0.61. In Ref. [39], the viscoelastic pipeline conveying fluid was simulated under the Boltzmann-Volterra integral model by means of the Bubnov-Galerkin method and the results showed that the oscillation amplitude differs much due to different $\alpha$. Compared with the results of Ref. [39], the numerical result in this part is verified to be correct.

In order to research whether the position changing influence the frequency response of maximum displacement and acceleration, the curves over the frequency interval $(100,120)$ for $x=0.96,0.98,1,1.02,1.04 \mathrm{~m}$ are shown in Figure 4(c) and Figure 4(d), It denotes that the curve still surges when $\omega$ only values $111 \mathrm{~Hz}$ and it is with tiny change for different position $x$. The above study indicates that the response of maximum displacement and acceleration can surge as variable fractional order and frequency changing, while the response changes subtly as position $x$ varying. In Ref.[40, a pipe conveying fluid was studied based on fourth-order Runge-Kutta integration algorithm. The displacement amplitude became larger near a certain frequency. Moreover, the displacement amplitude in Figure 4(c) is more sensitive to frequency and becomes larger near a certain frequency. Compared with the results of Ref. 40], the numerical result in this part is verified to be correct. 


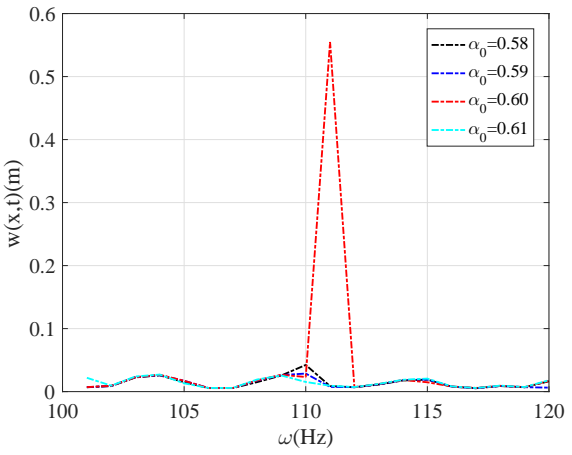

(a) Maximum displacement at different $\alpha_{0}$

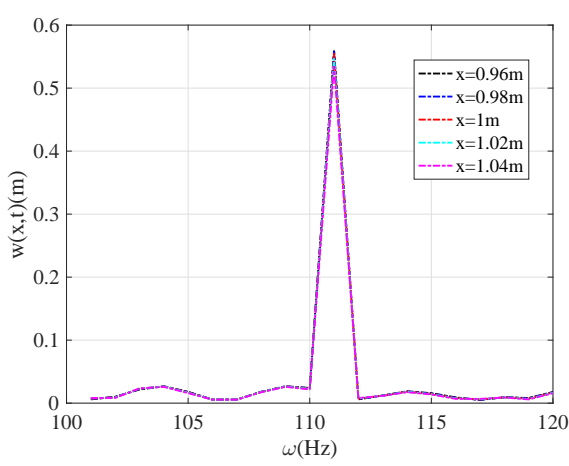

(c) Maximum displacement at different $\mathrm{x}$

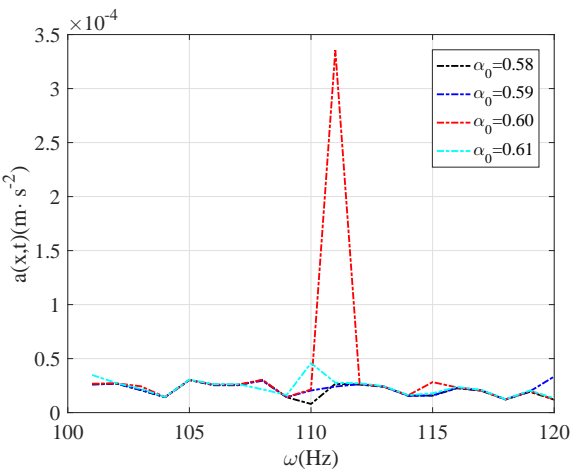

(b) Maximum acceleration at different $\alpha_{0}$

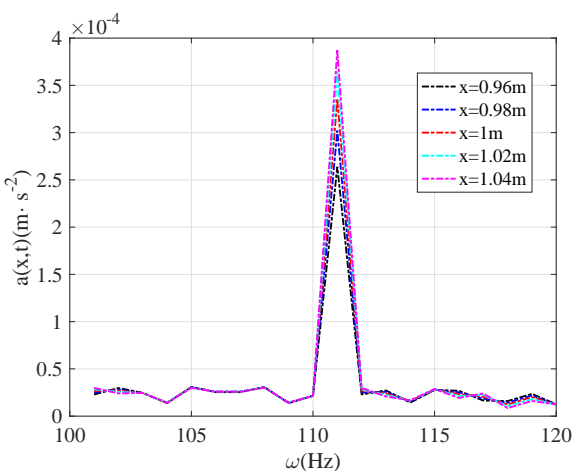

(d) Maximum acceleration at different $\mathrm{x}$

Figure 4: Dynamic response on viscoelastic pipes conveying fluid versus the frequency when $n$ values 6 for (a) Maximum displacement at different $\alpha_{0}$, (b) Maximum acceleration at different $\alpha_{0}\left(\alpha(t)=\alpha_{0}-|0.005-0.001 t|+0.005-\right.$ $0.001 t, x=1 \mathrm{~m}$ ) and for (c) Maximum displacement at different $\mathrm{x}$, (d) Maximum acceleration at different x. $(f(x, t)=100 \sin (\omega t) \mathrm{N} / \mathrm{m}, E=80 \mathrm{GPa}, \theta=0.01$, $\alpha(t)=0.6-|0.005-0.001 t|+0.005-0.001 t, V=5 \mathrm{~m} / \mathrm{s}, H=2 \mathrm{~m})$

The displacement, acceleration, strain and stress responses on viscoelastic pipes conveying fluid are investigated for different elastic modulus $E$ in this part. The parameters of the pipe are $f(x, t)=(100+100 \sin (200 t)) \mathrm{N} / \mathrm{m}, \theta=0.01$, $\alpha(t)=0.69-|0.005-0.001 t|+0.005-0.001 t, V=4.5 \mathrm{~m} / \mathrm{s}, H=10 \mathrm{~m}$. It can be 
seen that displacement $w(x, t)$, acceleration $a(x, t)$, strain $\varepsilon(x, t)$ are nonlinearly increasing and the nonlinear increasing amplitude becomes larger as elastic modulus decreasing linearly from Figure $5(\mathrm{a})$, Figure $5(\mathrm{~b})$ and Figure $5(\mathrm{c})$ However, stress $\sigma(x, t)$ shows the irregular behavior. The stress response for $E$ values $40 \mathrm{GPa}$ and $60 \mathrm{GPa}$ is nearly consistent from Figure $5(\mathrm{~d})$

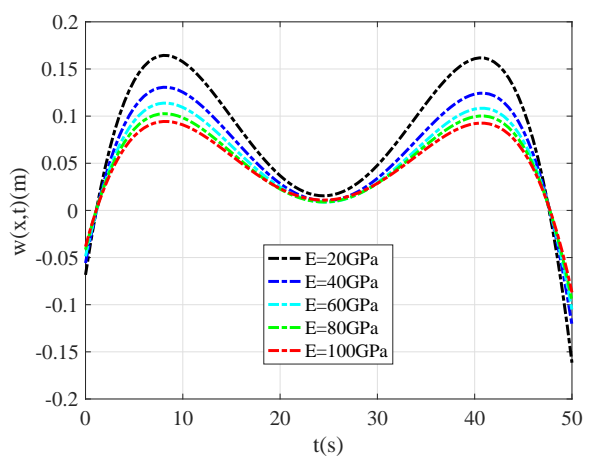

(a) Displacement

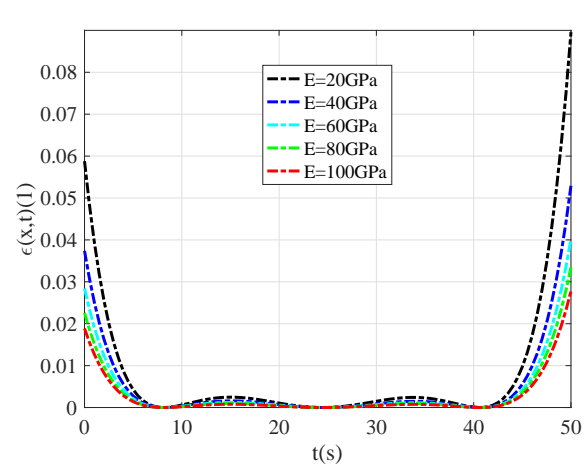

(c) Strain

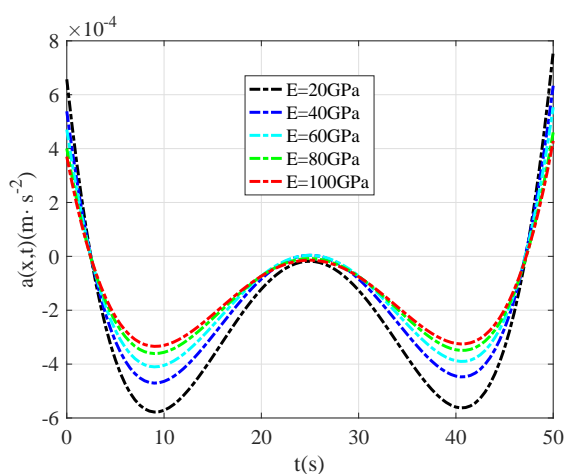

(b) Acceleration

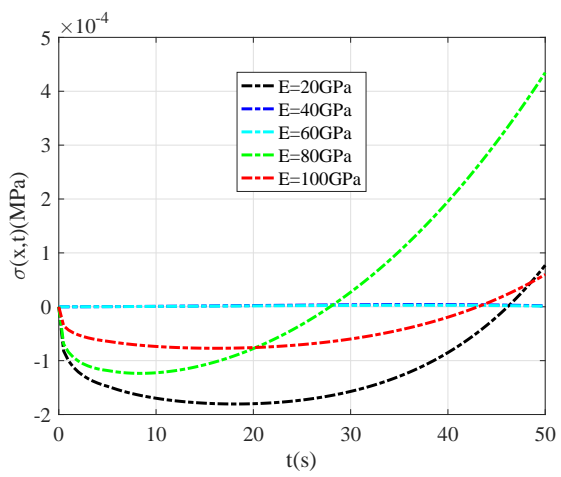

(d) Stress

Figure 5: Dynamic response on viscoelastic pipes conveying fluid versus the time at different modulus when $x$ values $0.5 H$ and $n$ values 6 for (a) Displacement, (b) Acceleration , (c) Strain and (d) Stress. $(f(x, t)=(100+100 \sin (200 t)) \mathrm{N} / \mathrm{m}$, $\theta=0.01, \alpha(t)=0.69-|0.005-0.001 t|+0.005-0.001 t, V=4.5 \mathrm{~m} / \mathrm{s}, H=10 \mathrm{~m})$

The displacement, acceleration, strain and stress responses on viscoelastic 
pipes conveying fluid are analyzed for different fluid velocities $V$ here. The parameters of the pipes are $f(x, t)=(100+100 \sin (200 t)) \mathrm{N} / \mathrm{m}, E=80 \mathrm{GPa}, \theta=$ $0.01, \alpha(t)=0.69-|0.005-0.001 t|+0.005-0.001 t, H=2 \mathrm{~m}$. In Ref. [41, a fluidconveying riser under integer order model was studied by means of finite element method. The governing equation of Ref. 41] can be considered as the governing equation (7) when $\alpha$ values 0 . The results in this paper indicate that the fluid velocity has little impact on displacement amplitude shown in Figure 6(a) and are similar to the results of Ref.41]. Compared with Ref. [41, the numerical result is verified to be correct. Also, it can be seen from Figure 6(a), Figure 6(b) and Figure 6(c) that the displacement $w(x, t)$, acceleration $a(x, t)$, strain $\varepsilon(x, t)$ are slightly increasing as fluid velocity increasing. And in Figure 6(d), the stress response with $t$ curve is a type of waves. The time to reach the crest is moving up as fluid velocity increasing overall. However, it is an exception that $V$ values $4.2 \mathrm{~m} / \mathrm{s}$.

The displacement, acceleration, strain and stress responses on viscoelastic pipes conveying fluid are considered for different lengths of pipes $H$ in this part. The parameters of the pipe are $f(x, t)=(100+100 \sin (200 t)) \mathrm{N} / \mathrm{m}, E=80 \mathrm{GPa}$, $\theta=0.01, \alpha(t)=0.69-|0.005-0.001 t|+0.005-0.001 t, V=4.5 \mathrm{~m} / \mathrm{s}, H=2 \mathrm{~m}$. It can be seen that the amplitude of displacement $w(x, t)$, acceleration $a(x, t)$, strain $\varepsilon(x, t)$ are increasing as the length $H$ increasing in Figure 7(a) Figure 7(b) and Figure $7(\mathrm{c})$, However, the amplitude of stress $\sigma(x, t)$ is increasing as $H$ increasing when $H$ is less than $5 \mathrm{~m}$, while stress $\sigma(x, t)$ shows irregular varying. The stress response isn't stable when $H$ becomes larger in Figure $7(\mathrm{~d})$.

The displacement, acceleration, strain and stress responses on viscoelastic pipes conveying fluid are studied for different amplitudes of force excitation here. The parameters of the pipe are $f(x, t)=(100+100 \sin (200 t)) \mathrm{N} / \mathrm{m}, E=80 \mathrm{GPa}$, $\theta=0.01, \alpha(t)=0.69-|0.005-0.001 t|+0.005-0.001 t, V=4 \mathrm{~m} / \mathrm{s}, H=2 \mathrm{~m}$. It can be seen that the amplitude of displacement $w(x, t)$, acceleration $a(x, t)$, strain $\varepsilon(x, t)$ are increasing as different magnitudes of force excitation increasing from Figure 8 . The viscoelastic pipes conveying fluid show linear changes as force excitation linearly changing. 
The above specific examples have proven the correctness of the proposed numerical computation scheme and numerical results according to the comparison with other methods. The dynamic properties of viscoelastic pipes conveying fluid are analyzed on the basis of Shifted Legendre Polynomials algorithm in the next part.

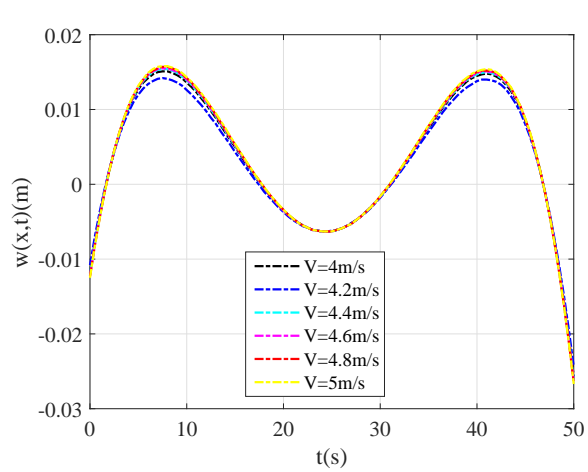

(a) Displacement

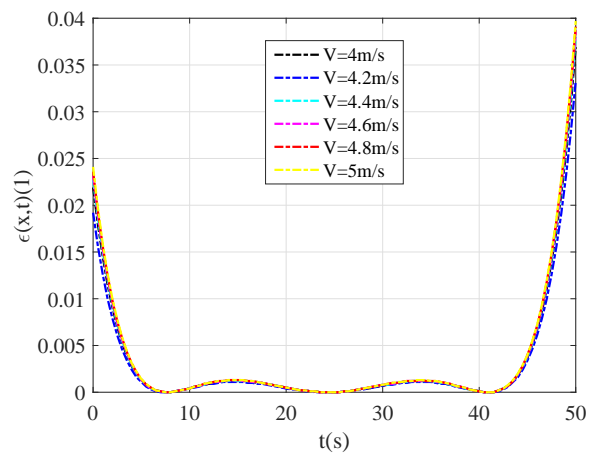

(c) Strain

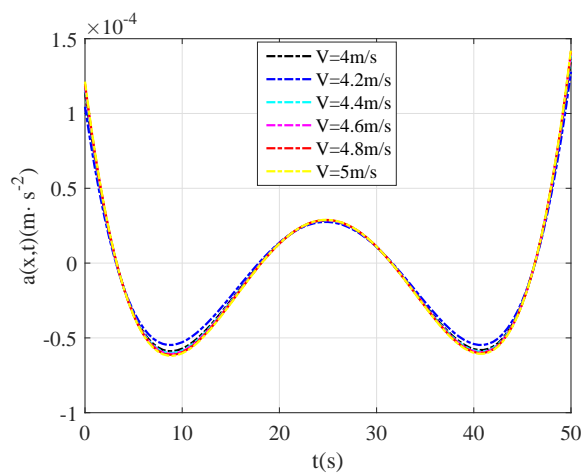

(b) Acceleration

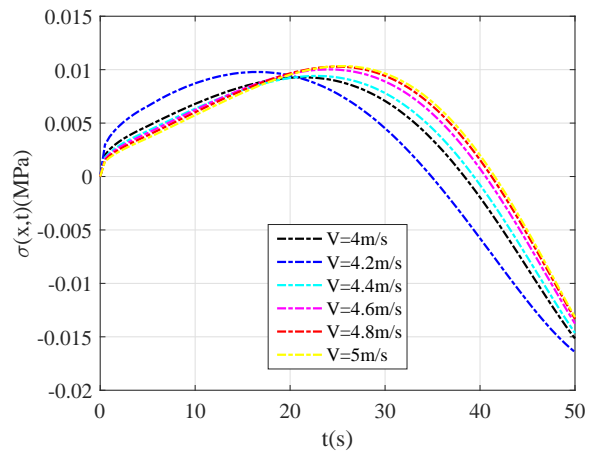

(d) Stress

Figure 6: Dynamic response on viscoelastic pipes conveying fluid versus the time at different velocities when $x$ values $0.5 H$ and $n$ values 6 for (a) Displacement, (b) Acceleration, (c) Strain and (d) Stress. $(f(x, t)=(100+100 \sin (200 t)) \mathrm{N} / \mathrm{m}$, $E=80 \mathrm{GPa}, \theta=0.01, \alpha(t)=0.69-|0.005-0.001 t|+0.005-0.001 t, H=2 \mathrm{~m})$ 


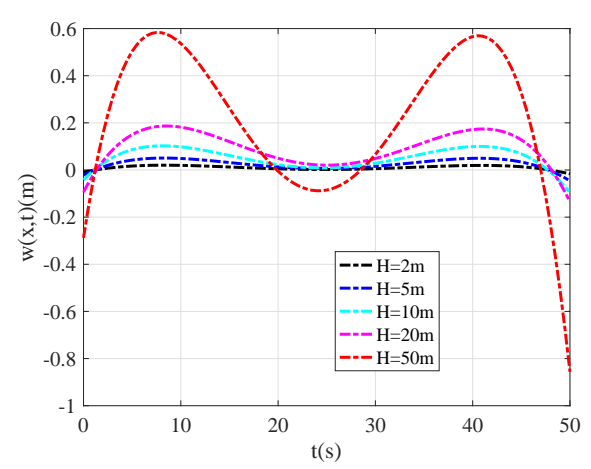

(a) Displacement

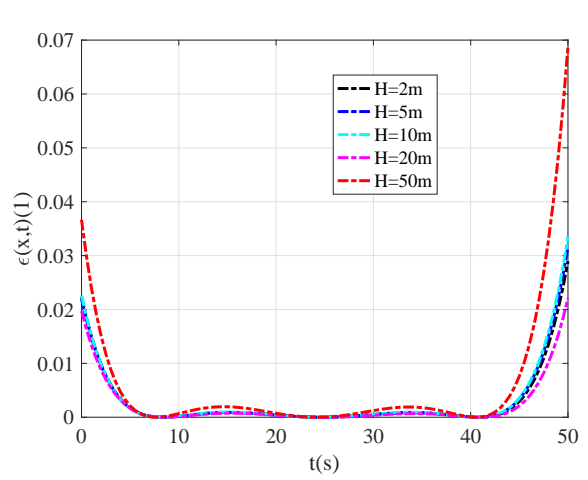

(c) Strain

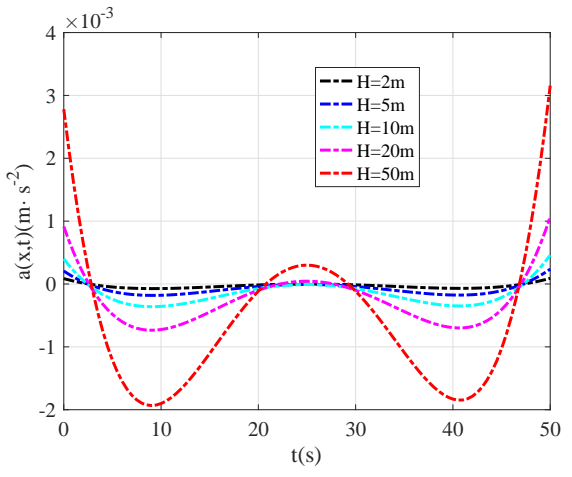

(b) Acceleration

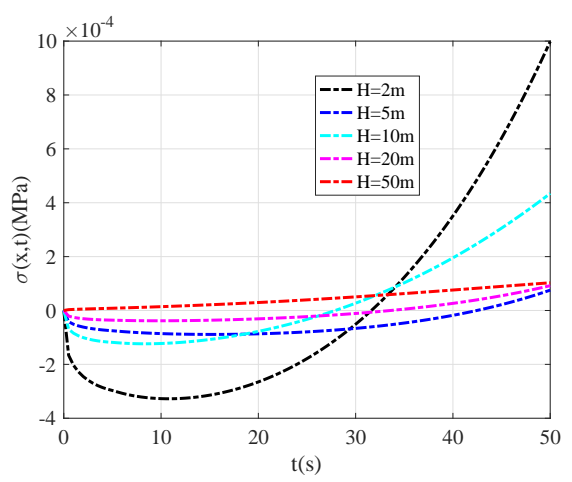

(d) Stress

Figure 7: Dynamic response on viscoelastic pipes conveying fluid versus the time at different lengths of pipes when $x$ values $0.5 H$ and $n$ values 6 for (a) Displacement, (b) Acceleration , (c) Strain and (d) Stress. $(f(x, t)=(100+$ $100 \sin (200 t)) \mathrm{N} / \mathrm{m}, E=80 \mathrm{GPa}, \theta=0.01, \alpha(t)=0.69-|0.005-0.001 t|+$ $0.005-0.001 t, V=4.5 \mathrm{~m} / \mathrm{s}, H=2 \mathrm{~m})$ 


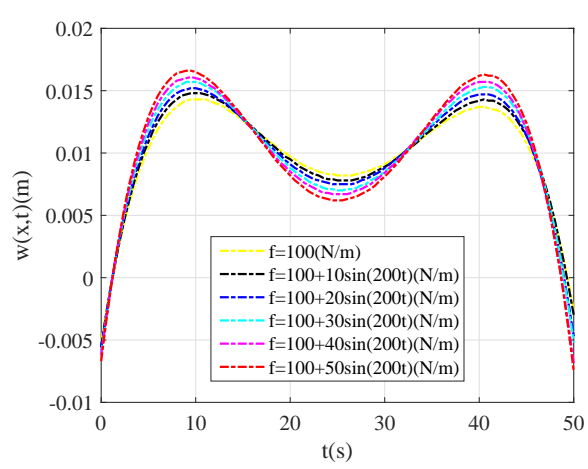

(a) Displacement

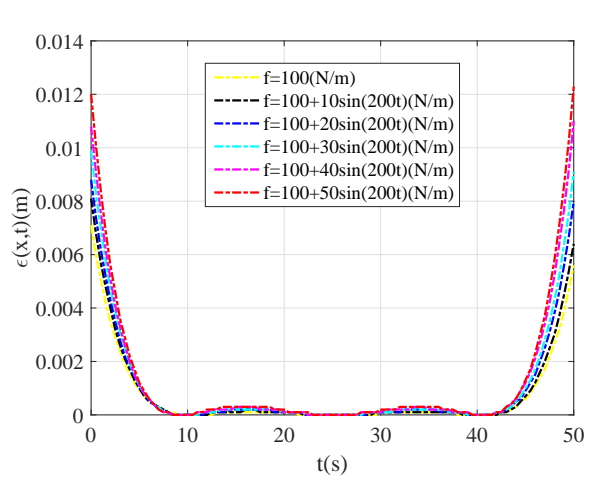

(c) Strain

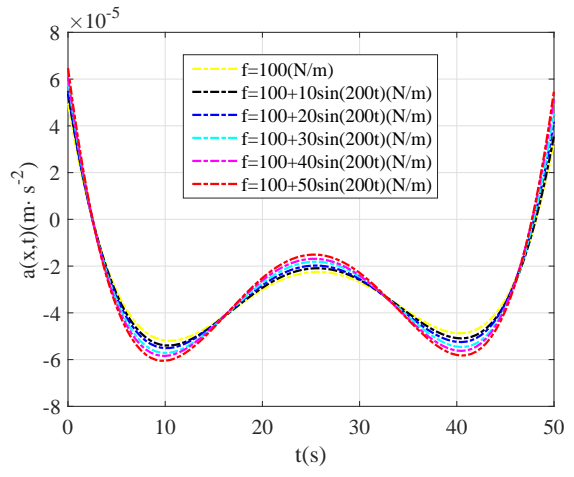

(b) Acceleration

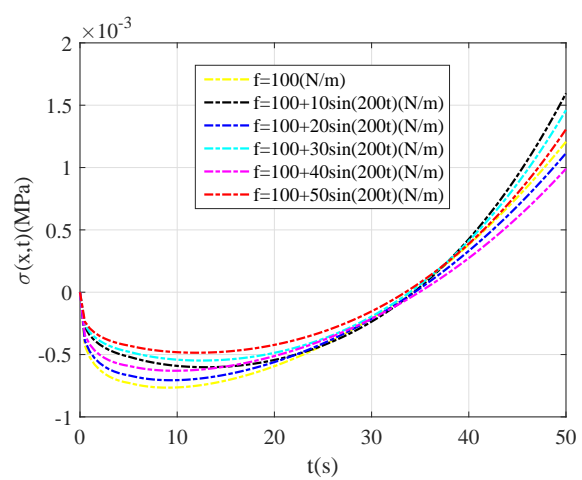

(d) Stress

Figure 8: Dynamic response on viscoelastic pipes conveying fluid versus the time at different force excitations when $x$ values $0.5 H$ and $n$ values 6 for (a) Displacement, (b) Acceleration , (c) Strain and (d) Stress. $(f(x, t)=(100+$ $100 \sin (200 t)) \mathrm{N} / \mathrm{m}, E=80 \mathrm{GPa}, \theta=0.01, \alpha(t)=0.69-|0.005-0.001 t|+$ $0.005-0.001 t, V=4 \mathrm{~m} / \mathrm{s}, H=2 \mathrm{~m})$

\subsection{Dynamic properties analysis}

In the above analysis, several nonlinear dynamic phenomenons have occurred. In order to further study the dynamic properties of viscoelastic pipes conveying fluid, the phase plane, Poincare surface of section and bifurcation diagram are selected to analysis the nonlinear behavior in this part. 
Considering a viscoelastic pipe conveying fluid where $f(x, t)=(100+500 \sin (20 t))$ $\mathrm{N} / \mathrm{m}, E=80 \mathrm{GPa}, \theta=0.01, \alpha(t)=0.6-|0.005-0.001 t|+0.005-0.001 t$, $V=5 \mathrm{~m} / \mathrm{s}, H=10 \mathrm{~m}$. The external force excitation is $(100+500 \sin (20 t)) \mathrm{N} / \mathrm{m}$. Then the excitation frequency is $20 \mathrm{~Hz}$. It can be noticed that the response time is 10 seconds in Figure 9(c). Within 10 seconds, 200 excitations is completed. It can be seen that there is no closed trajectory from Figure $9(\mathrm{a})$. Therefore there may be chaos motion in the system. There are uncountable points in Poincare surface of section which is shown in Figure $9(\mathrm{~b})$. Thus, there is chaos motion in the system.

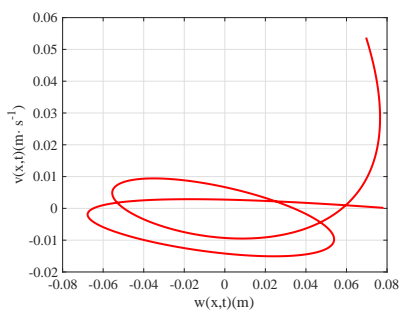

(a) Phase plane

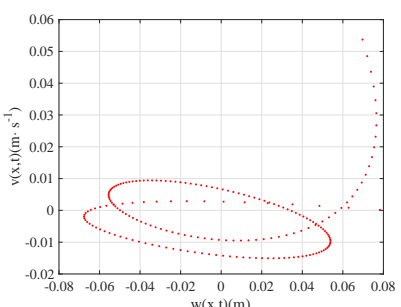

(b) Poincare surface of section

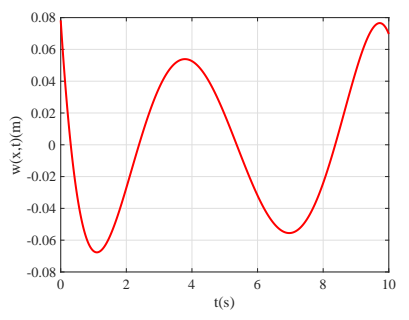

(c) Response time history

Figure 9: Dynamic analysis of viscoelastic pipes conveying fluid when $x$ values $0.5 H$ and $n$ values 6 for (a) Phase plane, (b) Poincare surface of section and (c) Response time history. $(f(x, t)=(100+500 \sin (20 t)) \mathrm{N} / \mathrm{m}, E=80 \mathrm{GPa}$, $\theta=0.01, \alpha(t)=0.6-|0.005-0.001 t|+0.005-0.001 t, V=5 \mathrm{~m} / \mathrm{s}, H=10 \mathrm{~m})$

Afterwards, three-dimensional bifurcation diagram is used to study the dynamic properties of viscoelastic pipes conveying fluid. As seen in Figure 10 the displacement $w(x, t)$ and the velocity $v(x, t)$ bifurcate while the acceleration is stable under $f(x, t)=(100+200 \sin (500 t)) \mathrm{N} / \mathrm{m}, E=80 \mathrm{GPa}, \theta=0.01$, $\alpha(t)=\alpha_{0}-|0.005-0.001 t|+0.005-0.001 t, V=5 \mathrm{~m} / \mathrm{s}, H=100 \mathrm{~m}$. It indicates variable fractional order $\alpha(t)$ won't change chaos state of $w(x, t)$ and $\alpha(x, t)$ in above conditions. However, position $x$ can change chaotic state of $w(x, t)$ and $v(x, t)$. The state is switched from chaos to stability as position $x$ varying. And it shows the most chaotic state in two ends of the pipes. 
The displacement $w(x, t)$, velocity $v(x, t)$ and acceleration $a(x, t)$ is chaos under $f(x, t)=(100+200 \sin (500 t)) \mathrm{N} / \mathrm{m}, E=80 \mathrm{GPa}, \theta=0.01, \alpha(t)=$ $0.5-|0.005-0.001 t|+0.005-0.001 t, H=10 \mathrm{~m}$ from Figure 11. It denotes the system begins more chaotic as fluid velocity increasing. Furthermore, the system is stable at the beginning of the pipe and chaotic at the end of the pipe.

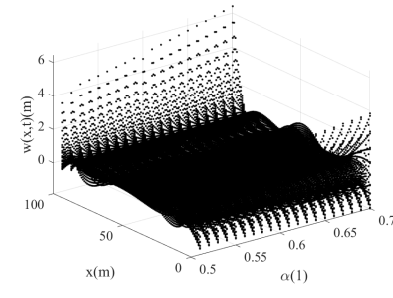

(a) Displacement

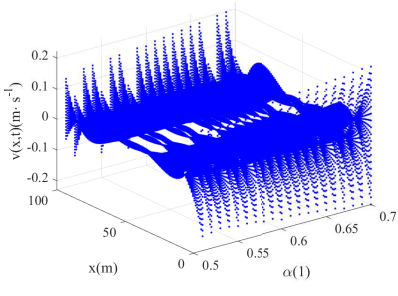

(b) Velocity

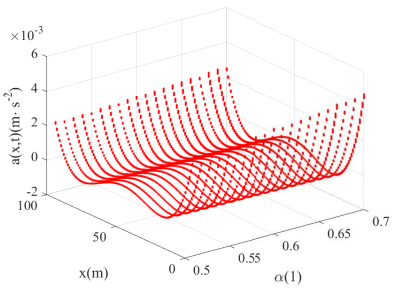

(c) Acceleration

Figure 10: Three-dimensional bifurcation diagram of viscoelastic pipes conveying fluid versus the position and the variable fractional order when $n$ values 6 for (a) Displacement, (b) Velocity and (c) Acceleration. $(f(x, t)=$ $(100+200 \sin (500 t)) \mathrm{N} / \mathrm{m}, E=80 \mathrm{GPa}, \theta=0.01, \alpha(t)=\alpha_{0}-|0.005-0.001 t|+$ $0.005-0.001 t, V=5 \mathrm{~m} / \mathrm{s}, H=100 \mathrm{~m})$

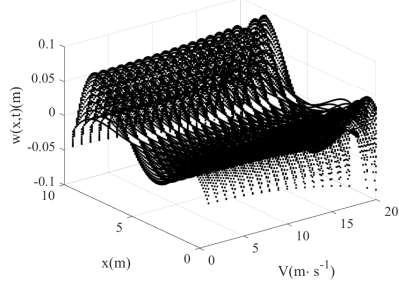

(a) Displacement

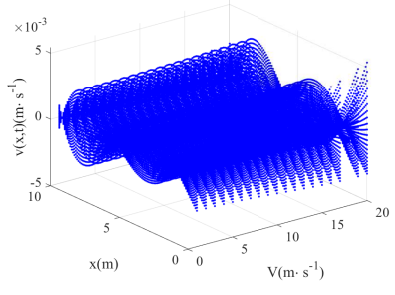

(b) Velocity

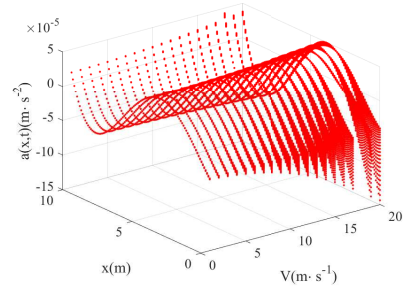

(c) Acceleration

Figure 11: Three-dimensional bifurcation diagram of viscoelastic pipes conveying fluid versus the position and the velocity when $n$ values 6 for (a) Displacement, (b) Velocity and (c) Acceleration. $(f(x, t)=(100+200 \sin (500 t)) \mathrm{N} / \mathrm{m}$, $E=80 \mathrm{GPa}, \theta=0.01, \alpha(t)=0.5-|0.005-0.001 t|+0.005-0.001 t, H=10 \mathrm{~m})$ 


\section{Conclusions}

As the study related to variable fractional nonlinear dynamic of fluid-solid interaction is very little, in the research, nonlinear dynamics for viscoelastic pipes conveying fluid is analyzed based on the variable fractional order model. A class of nonlinear variable fractional order integral-differential equation is firstly constructed as the more accurate governing equation compared with the previous ones. The Shifted Legendre Polynomials algorithm is presented to solve this type of equation. After being verified, this algorithm has the advantage of accuracy in dealing with this kind of nonlinear variable fractional order integraldifferential equation. The study yields the following conclusions from numerical results obtained by Shifted Legendre Polynomials algorithm:

(1) The amplitude response of viscoelastic pipes convey fluid differs too much with force excitation response due to the viscoelastic property.

(2) Displacement and acceleration of viscoelastic pipes conveying fluid will surge at a certain excitation frequency and variable fractional order. Their responses change slightly with position.

(3) Displacement, acceleration, strain of viscoelastic pipes convey fluid change regularly with elastic modulus, fluid velocity, the length of pipes and force excitation magnitude. However, the stress changes not completely regularly with these parameters.

(4) The system of viscoelastic pipes conveying fluid will be chaotic at some certain conditions. Chaotic motion won't be changed into stable state with the variable fractional order varying in a certain range. Stable state will be transformed into chaotic motion with the fluid velocity increasing. Especially this phenomenon occurs at the beginning of the pipe.

\section{Acknowledgements}

This work is supported by the Natural Science Foundation of Hebei Province (A2017203100) in China and the LE STUDIUM RESEARCH PROFESSORSHIP award of Centre-Val de Loire region in France. 


\section{References}

[1] G. Peng, Y.M. Xiong, L.M. Liu, Y. Gao, M.H. Wang, Z. Zhang, 3-D nonlinear dynamics of inclined pipe conveying fluid, supported at both ends, J. Sound Vib. 449 (2019) 405-426.

[2] G.H. Chang, Y. Modarres-Sadeghi, Flow-induced oscillations of a cantilevered pipe conveying fluid with base excitation, J. Sound Vib. 333 (2014) 4265-4280.

[3] F. He, H.L. Dai, Z.H. Huang, L. Wang, Nonlinear dynamics of a fluidconveying pipe under the combinedaction of cross-flow and top-end excitations, Appl. Ocean Res. 62 (2017) 199-209.

[4] M. Rahmati, H.R. Mirdamadi, S. Goli, Divergence instability of pipes conveying fluid with uncertain flow velocity, Physica A 491 (2018) 650-665.

[5] Y.F. Zhang, M.H. Yao, W. Zhang, B.C Wen, Dynamical modeling and multi-pulse chaotic dynamics of cantilevered pipe conveying pulsating fluid in parametric resonance, Aerospe. Sci. Technol. 68 (2017) 441-453.

[6] Y.L. Zhang, L.Q. Chen, External and internal resonances of the pipe conveying fluid in the supercritical regime, J. Sound Vib. 332 (2013) 2318-2337.

[7] L. Wang, Z.Y. Liu, A. Abdelkefi, Y.K. Wang, H.L. Dai, Nonlinear dynamics of cantilevered pipes conveying fluid: Towards a further understanding of the effect of loose constraints, Int. J. Non-Linear Mech. 95 (2017) 19-29.

[8] Y. Tang, Y.X. Zhen, B. Fang, Nonlinear vibration analysis of a fractional dynamic model for the viscoelastic pipe conveying fluid, Appl. Math. Model. 56 (2018) 123-136.

[9] D. Mitsotakis, D. Dutykh, Q. Li, E. Peach, On some model equations for pulsatile flow in viscoelastic vessels, Wave Motion 90 (2019) 139-151. 
[10] D. Yano, S. Ishikawa, K. Tanaka, S. Kijimoto, Vibration analysis of viscoelastic damping material attached to a cylindrical pipe by added mass and added damping, J. Sound Vib. 454 (2019) 14-31.

[11] S.A. Husain, R.S. Anderssen, Modelling the relaxation modulus of linear viscoelasticity using Kohlrausch functions, J. Non-Newtonian Fluid Mech. 125 (2005) 159-170.

[12] W. Yan, J. Ying, W.Q. Chen, The behavior of angle-ply laminated cylindrical shells with viscoelastic interfaces in cylindrical bending, Compos. Struct. 78 (2007) 551-559.

[13] C. Machiraju, A.V. Phan, A.W. Pearsall, S. Madanagopal, Viscoelastic studies of human subscapularis tendon: Relaxation test and a Wiechert model, Comput. Meth. Prog. Bio. 83 (2006) 29-33.

[14] Y. Peng, J.Z. Zhao, Y.M. Li, A wellbore creep model based on the fractional viscoelastic constitutive equation, Petrol. Explor. Develop. 44(6) (2017) 1038-1044.

[15] J.M. Long, R. Xiao, W. Chen, Fractional viscoelastic models with nonsingular kernels, Mech. Mater. 127 (2018) 55-64.

[16] M. Mokhtari, M.R. Permoon, H. Haddadpour, Aeroelastic analysis of sandwich cylinder with fractional viscoelastic core described by Zener model, J. Fluids Struct. 85 (2019) 1-16.

[17] Y. Yu, P. Perdikaris, G.E. Karniadakis, Fractional modeling of viscoelasticity in 3D cerebral arteries and aneurysms, J. Comput. Phys. 323 (2016) 219-242.

[18] R.F. Meng, D. Yin, C.S. Drapaca, A variable order fractional constitutive model of the viscoelastic behavior of polymers, Int. J. Non-Linear Mech. 113 (2019) 171-177. 
[19] Z. Li, H. Wang, R. Xiao, S. Yang, A variable-order fractional differential equation model of shape memory polymers, Chaos Solitons Fractals 102 (2017) 473-485.

[20] R.F. Meng, D. Yin, C.S. Drapaca, Variable-order fractional description of compression deformation of amorphous glassy polymers, Comput. Mech. 64 (2019) 163-171.

[21] Q.M. Al-Mdallal, M.I. Syam, The Chebyshev collocation-path following method for solving sixth-order Sturm-Liouville problems, Appl. Math. Comput. 232 (2014) 391-398.

[22] Q.M. Al-Mdallal, A.S.A. Omer, Fractional-order Legendre-collocation method for solving fractional initial value problems, Appl. Math. Comput. 321 (2018) 74-84.

[23] Q.M. Al-Mdallal, On fractional-Legendre spectral Galerkin method for fractional Sturm-Liouville problems, Chaos Solitons Fractals 116 (2018) 261267.

[24] M.H. Heydari, Z. Avazzadeh, M.F. Haromi, A wavelet approach for solving multi-term variable-order time fractional diffusion-wave equation, Appl. Math. Comput. 341 (2019) 215-228.

[25] Y.M. Chen, L.Q. Liu, B.F. Li, Y.N. Sun, Numerical solution for the variable order linear cable equation with Bernstein polynomials, Appl. Math. Comput. 238 (2014) 329-341.

[26] Y.M. Chen, L.Q. Liu, X. Li, Y.N. Sun, Numerical solution for the variable order time fractional diffusion equation with Bernstein polynomials, Comput. Model. Eng. Sci. 97(1) (2014) 81-100.

[27] Y.M. Chen, Y.Q. Wei, D.Y. Liu, H. Yu, Numerical solution for a class of nonlinear variable order fractional differential equations with Legendre wavelets, Appl. Math. Lett. 46 (2015) 83-88. 
[28] Y.M. Chen, L.Q. Liu, D.Y, Liu, D. Boutat, Numerical study of a class of variable order nonlinear fractional differential equation in terms of Bernstein polynomials, Ain Shams Eng. J. 9 (2018) 1235-1241.

[29] P. Assari, M. Dehghan, A meshless local Galerkin method for solving Volterra integral equations deduced from nonlinear fractional differential equations using the moving least squares technique, Appl. Numer. Math. 143 (2019) 276-299.

[30] J.S. Wang, L.Q. Liu, Y.M. Chen, L.C. Liu, D.Y. Liu, Numerical study for a class of variable order fractional integral-differential equation in terms of Bernstein polynomials, Comput. Model. Eng. Sci. 104(1) (2015) 69-85.

[31] B.P. Moghaddam, J.A.T. Machado, A computational approach for the solution of a class of variable-order fractional integro-differential equations with weakly singular kernels, B. Eng. Geol. Environ. 20(4) (2017) 1023-1042.

[32] Y.M. Chen, L.Q. Liu, B.F. Li, Y.N. Sun, Numerical solution for the variable order linear cable equation with Bernstein polynomials, Appl. Math. Comput. 238 (2014) 329-341.

[33] B.G. Sinir, D.D. Demir, The analysis of nonlinear vibrations of a pipe conveying an ideal fluid, Eur. J. Mech. B/Fluids 52 (2015) 38-44.

[34] Y.M. Chen, X.H. Ke, Y.Q. Wei, Numerical algorithm to solve system of nonlinear fractional differential equations based on wavelets method and the error analysis, Appl. Math. Comput. 251 (2015) 475-488.

[35] Y. Xu, Y.M. Zhang, J.J. Zhao, Error analysis of the Legendre-Gauss collocation methods for the nonlinear distributed-order fractional differential equation, Appl. Numer. Math. 142 (2019) 122-138.

[36] Y.X. Huang, Y. Zhao, T.S. Wang, H. Tian, A new Chebyshev spectral approach for vibration of in-plane functionally graded Mindlin plates with variable thickness, Appl. Math. Model. 74 (2019) 21-42. 
[37] Q.W. Ren, H.J. Tian, Numerical solution of the static beam problem by Bernoulli collocation method, Appl. Math. Model. 40 (2016) 8886-8897.

[38] S.M. Guo, L.Q. Mei, Z.Q. Zhang, J. Chen, Y. He, Y. Li, Finite difference/Hermite-Galerkin spectral method for multi-dimensional timefractional nonlinear reaction-diffusion equation in unbounded domains, Appl. Math. Model. 70 (2019) 246-263.

[39] B.A. Khudayarov, F.Zh. Turaev, Mathematical simulation of nonlinear oscillations of viscoelastic pipelines conveying fluid, Appl. Math. Model. 66 (2019) 662-679.

[40] Z.Y. Liu, L. Wang, X.P. Sun, Nonlinear forced vibration of cantilevered pipes conveying fluid, Acta Mech. Solida Sin. 31(1) (2018) 32-50.

[41] X.D. Zhang, R.Y. Gou, W.W. Yang, X.P. Chang, Vortex-induced vibration dynamics of a flexible fluid-conveying marine riser subjected to axial harmonic tension, J. Braz. Soc. Mech. Sci. 40 (2018) 365-377.

[42] Y.H. Wang, Y.M. Chen, Dynamic analysis of the viscoelastic pipeline conveying fluid with an improved variable fractional order model based on shifted Legendre polynomials, Fractal Fract. 3(4) (52) 2019, doi:10.3390/fractalfract3040052. 